\title{
Nanoscale
}

\begin{abstract}
Check for updates
Cite this: Nanoscale, 2018, 10, 5827

Received 19th December 2017,

Accepted 2nd February 2018

DOI: $10.1039 / c 7 n r 09436 a$

rsc.li/nanoscale

In this study we used $\mathbf{5 m}$ gold nanoparticles as delivery platforms to target cancer cells expressing the immune receptor Tim-3 using single chain antibodies. Gold surfaces were also covered with the cytotoxic drug rapamycin which was immobilised using a glutathione linker. These nanoconjugates allowed highly specific and efficient delivery of cytotoxic rapamycin into human malignant blood cells.
\end{abstract}

\section{Highly specific targeting of human acute myeloid leukaemia cells using pharmacologically active nanoconjugates $\uparrow$}

\author{
Inna M. Yasinska, ${ }^{\star a}$ Giacomo Ceccone, ${ }^{\mathrm{b}}$ Isaac Ojea-Jimenez, ${ }^{\mathrm{b}}$ Jessica Ponti, ${ }^{\mathrm{b}}$ \\ Rohanah Hussain, ${ }^{c}$ Giuliano Siligardi, ${ }^{c}$ Steffen M. Berger, ${ }^{d}$ Elizaveta Fasler-Kan, ${ }^{d}$ \\ Marco Bardelli, ${ }^{e}$ Luca Varani, ${ }^{e}$ Walter Fiedler, ${ }^{f}$ Jasmin Wellbrock, ${ }^{f}$ Ulrike Raap, ${ }^{9}$ \\ Bernhard F. Gibbs, ${ }^{{ }^{a}, \mathrm{~g}}$ Luigi Calzolai ${ }^{\mathrm{b}}$ and Vadim V. Sumbayev ${ }^{{ }^{a}}$
}

\section{Introduction}

Highly specific targeting of human malignant blood cells with the purpose of delivering cytotoxic drugs into them is a very promising but not a well-developed strategy for leukaemia treatment. Leukaemia is a blood/bone marrow cancer originating from self-renewing malignant immature precursors or transformed leukocytes. It is a severe and often fatal systemic malignancy which affects haematopoiesis, immune defence and followed by many other body systems. ${ }^{1}$ Chemotherapy of leukaemia is often problematic since the drugs used are generally toxic and could kill other growing and quickly proliferating cells such as stem cells, thus affecting normal haematopoiesis

${ }^{a}$ School of Pharmacy, University of Kent, Anson Building, Central Avenue, Chatham Maritime, Kent, ME4 4TB, UK. E-mail: I.Yasinska-24@kent.ac.uk, V.Sumbayev@kent.ac.uk, bernhard.gibbs@uni-oldenburg.de

${ }^{b}$ European Commission Joint Research Centre, Ispra, Italy.

E-mail: luigi.calzolai@ec.europa.eu

${ }^{c}$ Beamline B23, Diamond Light Source, Didcot, UK

${ }^{d}$ Department of Paediatric Surgery and Department of Biomedical Research, Children's Hospital, Inselspital, University of Bern, 3010 Bern, Switzerland ${ }^{e}$ Institute for Research in Biomedicine, Universita' della Svizzera italiana (USI), Via Vela 6, 6500 Bellinzona, Switzerland

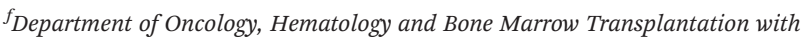
Section Pneumology, Hubertus Wald University Cancer Center,

University Medical Center Hamburg-Eppendorf, Germany

${ }^{g}$ Department of Dermatology, University of Oldenburg, Germany

$\dagger$ Electronic supplementary information (ESI) available. See DOI: 10.1039/ c7nr09436a and consequent recovery. ${ }^{2}$ Therefore, the use of drug delivery systems selectively targeting leukaemia cells could reduce overall toxicity and would be a considerable advantage in antileukaemia therapy.

We have recently shown that citrate-stabilised gold nanoparticles (AuNPs) can be used as drug delivery platforms. ${ }^{3,4}$ They display a reasonable level of biocompatibility, antiinflammatory properties and inability to affect cells on their own. ${ }^{3,5}$ In this study we designed a fundamentally novel type of nanocomplex, where $5 \mathrm{~nm}$ AuNPs were used as a delivery platform and carried a $27 \mathrm{kDa}$ single chain antibody against $\mathrm{T}$ cell immunoglobulin and mucin domain 3 (Tim-3) at a ratio of 1 AuNP : 1 antibody molecule. The rest of the gold surface was covered with rapamycin immobilised in the form of glutathione-SH ester.

The immune receptor Tim-3 is highly expressed in human acute myeloid leukaemia (AML) cells compared to healthy leukocytes and thus can be used as a receptor for targeted drug-delivery complexes. ${ }^{6-8}$ Rapamycin inhibits the activity of a mammalian target of rapamycin (mTOR), an enzyme displaying serine/threonine protein kinase activity which acts as a master regulator of translational pathways in AML cells. Inhibiting mTOR leads to rapid killing of AML cells. ${ }^{3,8}$ Using these nanoconjugates (NCJ) we managed to successfully deliver rapamycin into AML cells and reduce mTOR activity. The concentration of rapamycin required to reach such an effect was at least 50 times lower compared to that of free rapamycin required to achieve a similar effect. We therefore concluded that our technology is of potential use for highly specific targeting of AML cells.

\section{Materials and methods}

\section{Materials}

RPMI-1640 medium, foetal bovine serum and supplements and basic laboratory chemicals were purchased from Sigma 
(Suffolk, UK). Maxisorp ${ }^{\mathrm{TM}}$ microtitre plates were provided either by Nunc (Roskilde, Denmark) or Oxley Hughes Ltd (London, UK). Antibodies against phospho-S65 and total eIF4E-BP were obtained from Cell Signaling Technology (Danvers, MA USA). Goat anti-mouse and goat anti-rabbit fluorescence dye-labelled antibodies were obtained from Li-Cor (Lincoln, Nebraska USA). The anti-Tim-3 mouse monoclonal antibody and its single chain variant were produced as previously described. ${ }^{9}$ All other chemicals purchased were of the highest grade of purity and available commercially from Fisher Scientific (Loughborough, UK) and Sigma (Suffolk, UK).

\section{Gold nanoparticles}

The synthesis of $5 \mathrm{~nm}$ AuNPs was performed essentially as described previously. ${ }^{10}$ Briefly, $5 \mathrm{ml}$ of aqueous gold(III) chloride trihydrate $(10 \mathrm{mM})$ and $2.5 \mathrm{ml}$ of aqueous sodium citrate (100 $\mathrm{mM})$ were added to $95 \mathrm{ml}$ of MilliQ-water in a round bottom flask equipped with a magnetic stirrer, and the resulting pale yellow solution was allowed to cool down to $1-2{ }^{\circ} \mathrm{C}$. Under vigorous stirring $1 \mathrm{ml}$ of chilled $\left(4^{\circ} \mathrm{C}\right)$ aqueous sodium borohydride $(0.1 \mathrm{M})$ was added and the resulting dark red solution was then stirred for further $10 \mathrm{~min}$ in an ice bath before being allowed to warm to the room temperature.

Nanomaterials were characterised as described before. ${ }^{3}$

\section{Cells}

THP-1 human leukaemia monocytic macrophages were purchased from the European collection of Cell Cultures (Salisbury, UK). They were grown in RPMI 1640 media supplemented with $10 \%$ foetal calf serum, penicillin (50 IU ml ${ }^{-1}$ ) and streptomycin sulphate $\left(50 \mu \mathrm{g} \mathrm{ml} \mathrm{m}^{-1}\right)$ and used for the experiments during passages 8-20.

Primary human AML cells were obtained from the sample bank of the University Medical Centre Hamburg-Eppendorf (Ethik-Kommission der Ärztekammer Hamburg, reference: PV3469). The cells were cultured in IMDM medium containing 15\% BIT 9500 serum substitute, $100 \mu \mathrm{M}$ mercaptoethanol, 100 $\mathrm{ng} \mathrm{ml}^{-1}$ stem cell factor (SCF), $50 \mathrm{ng} \mathrm{ml}^{-1}$ FLT3 ligand, 20 $\mathrm{ng} \mathrm{ml}^{-1}$ G-CSF, $20 \mathrm{ng} \mathrm{ml}^{-1}$ IL-3, $1 \mu \mathrm{M}$ UM729 and $500 \mathrm{nM}$ stemregenin 1 (SR1) as described before. ${ }^{11}$

\section{Western blot analysis}

Phosphorylation of the mTOR substrate - eukaryotic initiation factor $4 \mathrm{E}$ binding protein (eIF4E-BP) in the position serine 65 (S65) was analysed by western blot analysis as described previously. ${ }^{8}$ Actin expressions were measured in order to validate equal protein loading. Li-Cor (Lincoln, Nebraska USA) goat secondary antibodies conjugated with fluorescent dyes for $60 \mathrm{~min}$ were employed according to the manufacturer's protocol and proteins were visualised using a Li-Cor Odyssey imaging system. The western blot data were subjected to quantitative analysis using Odyssey software determining the levels of mTOR-dependent phosphorylation of eIF4e-BP. ${ }^{8}$
Characterisation of the presence of a cell surface of Tim-3 and its natural ligand/trafficking partner galectin-9

Cell surface expression of Tim-3 and its natural ligand galectin-9 were analysed using fluorescence-activated cell sorting (FACS) as previously described. ${ }^{12}$ Briefly, THP-1 cells were incubated with PMA to increase the expression of the Tim-3/Gal-9 complex or left untreated. The cells were then fixed with freshly prepared $2 \%$ paraformaldehyde, washed $3 \times$ with PBS, and permeabilised with $0.1 \%$ TX-100. In a control set of experiments the THP-1 cells were not fixed/permeabilized. All the cells were stained with fluorochrome-labelled antibodies for 1 hour at room temperature and analysed using a FACS Calibur cytometer with CellQuestPro software (Becton Dickinson, USA). We also performed a Li-Cor on cell assay (also known as in cell assay, ICA) in order to stain cell surfacebased Tim-3. In cell western (ICW) analysis using methanol as a cell permeabilisation agent was employed to monitor differences between Tim-3 localised inside and outside the cell. Both assays were described previously.

\section{Synchrotron radiation circular dichroism spectroscopy}

Nanomaterials were characterised at the B23 beamline for synchrotron radiation circular dichroism (SRCD), Diamond Light Source (Didcot, UK). SRCD measurements were performed using a $10 \mathrm{~cm}$ path length cell, $3 \mathrm{~mm}$ aperture diameter and $800 \mu \mathrm{l}$ capacity using Module B with a $1 \mathrm{~nm}$ increment, $1 \mathrm{~s}$ integration time, and $1.2 \mathrm{~nm}$ bandwidth at $23{ }^{\circ} \mathrm{C} .{ }^{13,14}$ The results obtained were processed using the CDApps programme $^{13-15}$ and Origin software (OriginLab ${ }^{\mathrm{TM}}$ ).

\section{Transmission electron microscopy (TEM) and dynamic light scattering (DLS)}

The NCJ suspension $(3 \mu \mathrm{l})$ was manually spotted, after 2 min of sonication, on pre-treated (glow discharge, LEICA EM ACE200, 10 mA-30 s) Formvar Carbon coated 200 mesh copper grids (Agar Scientific, USA). Samples were then dried overnight in a desiccator. Transmission electron microscopy (TEM-JEOL JEM 2100, JEOL-Italy) coupled with energy dispersive X-ray spectroscopy (EDX; X-Flash Detector 5030, Brüker-Italy) was used for imaging and elemental analysis at $120 \mathrm{kV}$. Quantitative analysis was obtained by the Cliff-Lorimer model using QUANTAX 200 software (Brüker, Italy). The results were expressed as mass \% of the whole sample measured in the analysed image and compared with NCJ precursors.

The average size in the solution of the nanoconjugate was also assessed by DLS (Zetasizer Nano Zs, Malvern Instruments; Malvern, UK). ${ }^{16}$

\section{Statistical analysis}

Each experiment was performed at least three times and statistical analysis when comparing two events at a time was conducted using a two-tailed Student's $t$-test. Statistical probabilities $(p)$ were expressed as ${ }^{*}$, where $p<0.05,{ }^{* *} p<0.01$ and ${ }^{* * *} p<0.001$. 


\section{Results and discussion}

\section{Generation and characterisation of nanoconjugates}

The anti-Tim-3 single-chain antibody (anti-Tim-3-ScAb) was coupled to $5 \mathrm{~nm}$ AuNPs using glutathione (GSH, tripeptide containing $\gamma$-glutamate, cysteine-SH and glycine) as a linker. A $0.05 \mathrm{M}$ solution of GSH was incubated for $2 \mathrm{~h}$ at room temperature with a 1:1 mixture of water solutions of $0.4 \mathrm{M}$ 1-ethyl-3-(3-dimethylpropyl)-carbodiimide (EDC) and $0.1 \mathrm{M}$ $N$-hydroxysuccinimide (NHS) to allow the activation of $-\mathrm{COOH}$ groups of the GSH thus enabling them to interact with the amino groups of the anti-Tim-3-ScAb. Activated GSH was then mixed with anti-Tim-3-ScAb in an equimolar ratio and incubated for at least $2 \mathrm{~h}$ at $37^{\circ} \mathrm{C}$. The antibody was then mixed with $5 \mathrm{~nm}$ AuNPs (0.5 mM) at a ratio of 1 AuNP : 1 antibody molecule. This mixture was incubated for at least $18 \mathrm{~h}$ at room temperature.

The available amount of AuNPs was calculated using the following formula:

$$
\begin{aligned}
& N_{\mathrm{AuNP}}=m_{\mathrm{total} \mathrm{Au}} / m_{\mathrm{AuNP}} \quad(m=\text { mass; } N=\text { quantity }) ; \\
& m_{\text {total Au }}=C_{\mathrm{Au}}\left(\mathrm{mol} \mathrm{l}^{-1}\right) \times \mathrm{MW}_{\mathrm{Au}}\left(\mathrm{g} \mathrm{mol}^{-1}\right) \times V_{\mathrm{AuNP}}(\mathrm{l})
\end{aligned}
$$

( $C=$ molar concentration, $\mathrm{MW}=$ molecular weight,$V_{\mathrm{AuNP}}=$ the volume of the AuNP suspension used);

$$
\begin{gathered}
m_{1 \mathrm{AuNP}}=\rho_{\mathrm{Au}} \times V_{1 \mathrm{AuNP}} ; \\
\left(\rho=\text { density }, V=\text { volume} ; \rho_{\mathrm{Au}}=19.3 \mathrm{~g} \mathrm{~cm}^{-3}\right) ; \\
V_{1 \mathrm{AuNP}}=4 / 3 \pi R^{3} ; \quad(R=\text { radius of a AuNP })
\end{gathered}
$$

$R=d_{1 \mathrm{AuNP}} / 2 ; \quad(d=$ diameter of 1 AuNP expressed in $\mathrm{cm})$.

The amount of antibody molecules was calculated from the molar weight of the anti-Tim-3-ScAb (27000 $\left.\mathrm{g} \mathrm{mol}^{-1}\right)$ and the Avogadro constant so that 1 mole of anti-Tim-3-ScAb contained $6.02 \times 10^{23}$ antibody molecules.

Following antibody immobilisation, the remaining gold surface of each particle was covered with molecules of rapamycin esterified with the GSH ester prepared as previously described $^{4}$ so that 1 AuNP carried a maximum of 1000 rapamycin molecules. The size of AuNPs ( $5 \mathrm{~nm}$ ) was confirmed using DLS. The size of the NCJ was approximately $10.8 \mathrm{~nm}$.

Materials were characterised using SRCD spectroscopy measuring both near-UV and far-UV spectra. HGS-CO-antiTim-3-ScAb and HSG-CO-rapamycin (HGS-CO is the glutathione residue), or a mixture of both, AuNPs carrying either anti-Tim-3-ScAb alone or rapamycin were characterised as controls (Fig. 1). We found that both near-UV and far-UV NCJ (NCJ is the same as that represented in Fig. 5 and 7 containing rapamycin and anti-Tim-3-ScAb [1000:1] per nanoparticle) displayed spectral profiles that were different from those of the controls suggesting that the generation of the complexes was successful with different conformational behaviours (Fig. 2).
A

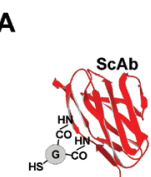

D

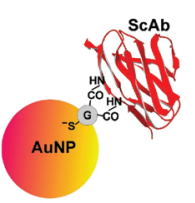

B

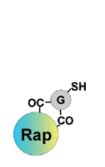

$\mathbf{E}$

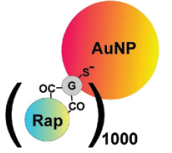

C
$\mathbf{F}$

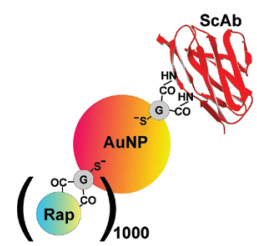

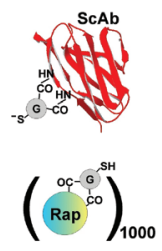

Fig. 1 Nanoconjugates used for the characterisation of biological tests. Six types of materials were generated: (A) glutathione GSH-activated anti-Tim-3-ScAb, (B) glutathione GSH activated rapamycin, (C) a mixture of $A$ and $B$ at a ratio of $1: 1000$, (D) $5 \mathrm{~nm}$ AuNPs carrying anti-Tim-3$\mathrm{ScAb},(\mathrm{E}) 5 \mathrm{~nm}$ AuNPs carrying rapamycin and (F) nanoconjugate containing $5 \mathrm{~nm}$ AuNPs coupled with anti-Tim-3-ScAb and 1000 rapamycin via GSH.

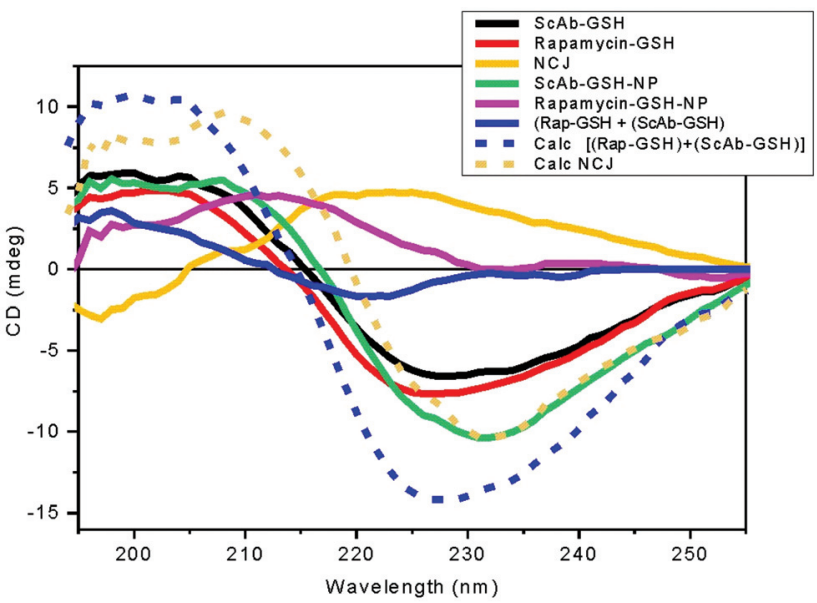

Fig. 2 Observed far-UV SRCD spectra of ScAb-GSH (black), ScAbGSH-NP (green), nanoconjugate NCJ (rapamycin-GSH and ScAb-GSH covalently attached to Au nanoparticles [1000:1:1]) (yellow), rapamycin-GSH (red), rapamycin-GSH-NP (magenta), a mixture of rapamycinGSH with ScAb-GSH (blue), the calculated spectrum of the mixture of rapamycin-GSH and ScAb-GSH (dashed blue) simulated by adding the spectrum of rapamycin-GSH (red) to that of ScAb-GSH (black) and calculated NCJ (dashed yellow) simulated by adding the spectrum of ScAbGSH-NP (green) to that of rapamycin-GSH-NP (magenta). Each observed spectrum was the average of 4 consecutive repeated scans.

\section{Characterisation of nanoparticle construct conformations}

In the far-UV region (190-250 $\mathrm{nm}$ ), the SRCD spectrum of the single chain antibody-GSH (ScAb-GSH) showed a negative band at $230 \mathrm{~nm}$ and a positive band at $205 \mathrm{~nm}$ (Fig. 2, black) indicating significant $\beta$-strand conformation. In particular, the smaller intensity of the positive band was indicative of the presence of two types of handedness of the $\beta$-strand backbone conformation of the single chains. Here, a small excess of the 
right-handed twist was observed, which is characterised by the bisignate shape with a negative band in the 215-230 nm region and a positive band in the 195-205 $\mathrm{nm}$ region. This interpretation appears to be consistent with the $\beta$-strand conformation content estimation of about $45 \%$, of which $18 \%$ was left-handed and $27 \%$ right-handed using the BestSel algorithm. ${ }^{16}$ The single chain antibody-GSH covalently attached to a gold nanoparticle (ScAb-GSH-NP, Fig. 2, green) retained the $\beta$-strand type of SRCD spectrum observed without the gold nanoparticle but with a slight reduction of the left-handed $\beta$-strand content.

The stability of the nanoconjugates was tested by incubating the materials at increasing temperatures and $\mathrm{pH}$ levels. This was followed by spectrophotometric detection of reduced glutathione using 5,5'-dithiobis-(2-nitrobenzoic acid) ${ }^{17}$ and antibody detection using western blotting (to see if these components were displaced from the gold surface). We did not observe detectable amounts of either GSH (data not shown) or antibody under any of the conditions tested (ESI Fig. $1 \dagger$ ).

We also used TEM to characterise the nanoconjugates. We observed that NCJ particles show spherical morphology typical of AuNPs with a primary size diameter (with a short axis length of $5.85 \mathrm{~nm}$ ). Lower electron-dense zones, compared to gold, were both around single particles and aggregates/ agglomerates (indicated by arrows in ESI Fig. $2 \dagger$ ) could be due to AuNP-bound antibodies. EDX analysis shows that the percentage of sulfur is practically negligible in the AuNP alone $(0.89 \%)$, while it increases (as expected) in the AuNP-Ab complex (2.28\%) and in the NCJ sample (5.54\%). These results fit qualitatively well with expected outcomes. In fact, both the anti-Tim-3 antibody and rapamycin were coupled to AuNPs using glutathione that contains a cysteine amino acid with an -SH terminal group. Overall, the data show that both the antibody and rapamycin are bound to AuNPs (ESI Fig. $3 \dagger$ ).

The DLS data for the NCJ sample give a $Z$-average of around $2 \mu \mathrm{m}$ and a polydispersity index of 0.39 . These results indicate that in solution the sample is polydispersed. As shown previously $^{18}$ in DLS the presence of a few agglomerates could cover the signal of smaller particles present in solution. This interpretation is consistent with the TEM results (ESI Fig. $1 \dagger$ ) which show the presence of a large number of small AuNP conjugates together with the presence of larger agglomerates.

It has been reported that several antibody molecules can be successfully immobilised on a single particle. ${ }^{19}$ In our case, a one to one ratio was optimal to ensure that there was sufficient space on the AuNP surface to be covered with rapamycin.

The SRCD spectrum of rapamycin-GSH showed qualitatively a $\beta$-strand type of shape (Fig. 2, red), a negative CD band at about $225 \mathrm{~nm}$ and a positive band at about $205 \mathrm{~nm}$. This may reflect the conformation of the cysteinyl-glycine dipeptide chromophore, which was too small in terms of intensity to be analysed with BestSel accurately. Upon attachment to the gold nanoparticle (NP), rapamycin-GSH-NP (Fig. 2 magenta) revealed a significant spectral change showing only a positive band at $215 \mathrm{~nm}$ with the disappearance of the negative band at $225 \mathrm{~nm}$. These SRCD changes appear to be dominated by the $\pi-\pi^{*}$ transitions of the amide bonds though the contribution from the trienyl group sensitive to the conformational changes of the flexible rapamycin skeleton backbone which cannot be ruled out.

It is interesting to note that the SRCD spectrum of the mixture of rapamycin-GSH and ScAb-GSH [(Rap-GSH) + (ScAbGSH), Fig. 2, blue] was not superimposable to that calculated by adding the spectrum of rapamycin-GSH to the one of ScAbGSH (Calc. [(Rap-GSH) + (ScAb-GSH)], Fig. 2, dashed blue), suggesting binding interactions between rapamycin-GSH and ScAb-GSH. Similarly, the SRCD changes between the observed spectrum of the NCJ (Fig. 2, yellow) and the one calculated by adding the spectrum of rapamycin-GSH-NP to that of ScAbGSH-NP (Calc-NCJ-NP, Fig. 2, dashed yellow) highlighted another conformational behaviour of the ScAb-GSH and rapamycin-GSH when coupled to the same nanoparticle. As for rapamycin-GSH with and without coupling to the gold nanoparticle, the BestSel secondary structure content estimation of the mixture [(Rapa-GSH) + (ScAb-GSH)] and NCJ from SRCD spectra were not sufficiently accurate to be considered.

The characterisation by SRCD spectroscopy of the samples and in particular NCJ that appear to be active against primary leukaemia cells is also important for quality control since the SRCD spectra can be used as a fingerprint of these constructs.

\section{Undifferentiated and differentiated THP-1 human AML cells have different amounts of surface-based Tim-3}

THP-1 human myeloid leukaemia cells express Tim-3 which acts as a receptor and a trafficker for galectin-9, a protein which protects AML cells against host immune defence. ${ }^{6,20}$ Using FACS (Fig. 3) we found that undifferentiated THP-1 cells have much less surface-based Tim-3 and galectin-9, respectively, than the cells exposed for $24 \mathrm{~h}$ to $100 \mathrm{nM}$ phorbol 12-myristate 13-acetate (PMA). In undifferentiated cells, surface-based Tim-3 molecules are occupied with galectin-9 and can only be clearly detected using on cell assay where the cells were pre-incubated with the anti-Tim-3 antibody for $2 \mathrm{~h}$ that enables the displacement of galectin-9 (Fig. 4). PMA activates protein kinase $\mathrm{C}$ alpha (PKC $\alpha$ ), an enzyme that triggers the exocytosis of Tim-3 and the secretion of galectin-9, thus increasing the amount of both proteins on the cell surface. However, both undifferentiated and differentiated THP-1 cells express high amounts of Tim-3 and most of these proteins were observed to translocate onto the cell surface upon the stimulation of the cells with PMA as detected by the on cell assay and in cell western analysis (Fig. 4). Some of the Tim-3 molecules were bound to galectin-9, others were present in their free form and thus became clearly detectable by both FACS and the on cell assay/in cell western analysis (Fig. 3 and 4).

We exposed the resting undifferentiated THP-1 cells for $2 \mathrm{~h}$ at $37{ }^{\circ} \mathrm{C}$ to $100 \mathrm{ng} \mathrm{ml}^{-1}$ human stem cell factor (SCF) that triggers mTOR activation and causes a non-significant increase in the level of cell surface-based Tim-3 in THP-1 cells. Exposures were performed with and without $1 \mathrm{~h}$ pre-treatment with NCJ (allowing for a maximal possible concentration of rapamycin 

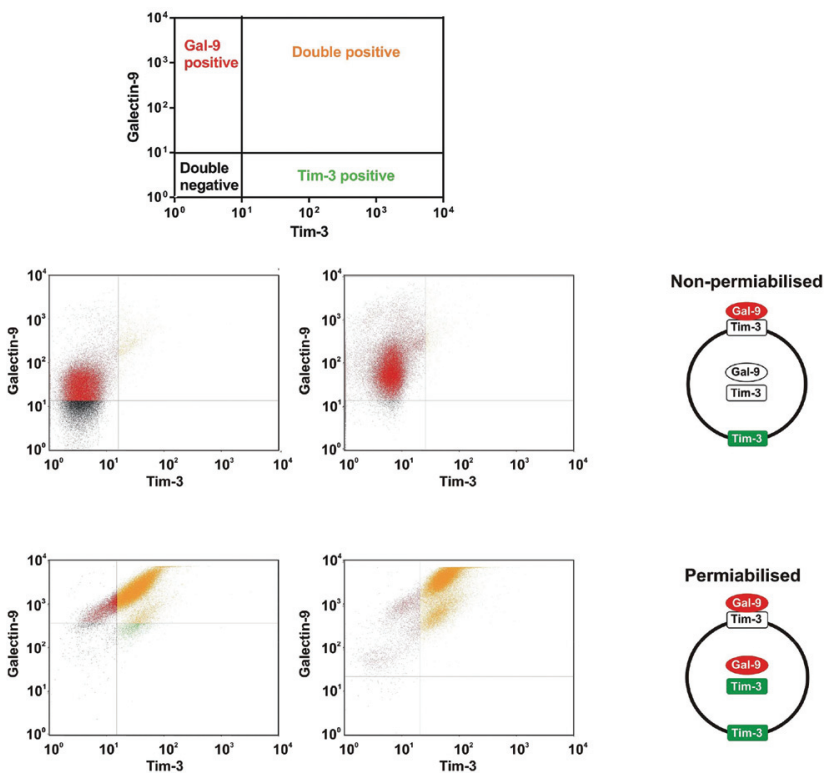

Fig. 3 Surface-based and total (intracellular + surface-based) Tim-3 and its ligand galectin-9 (carried by Tim-3) in THP-1 cells. Non-treated THP-1 cells (left two panels) and those treated for $24 \mathrm{~h}$ with $100 \mathrm{nM}$ PMA (right two panels) were subjected to the measurement of surfacebased and total (cells were permeabilised using methanol) Tim-3 and galectin- 9 by FACS as described in the Materials and methods section. Each experiment was performed at least three times. Images are from one experiment representative of three which gave similar results.
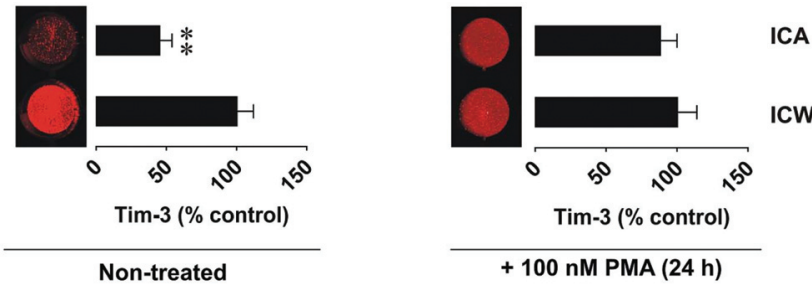

Fig. 4 Tim-3 is mostly located inside resting THP-1 cells but is externalised after PMA-dependent PKC $\alpha$ activation. Tim- 3 was measured using a Li-Cor on cell assay with (in cell western) or without (on cell assay) methanol permeabilisation (see the Materials and methods section). Exposure to the anti-Tim-3 antibody was performed for $2 \mathrm{~h}$. Images are from one experiment representative of five which gave similar results. Data show mean values \pm SEM of five independent experiments; ${ }^{* *} p<0.01$.

of $200 \mathrm{nM}$ - given the ideal case when each AuNP coupled with anti-Tim-3-ScAb carries a maximum of 1000 rapamycin molecules (illustrated in Fig. 5)). We found that nanoconjugates were able to abrogate SCF-induced mTOR-dependent eIF4E-BP phosphorylation but did not impact on background S65phospho-eIF4E-BP levels (Fig. 6). Neither of the treatments affected cell viability.

We then asked whether an increase in Tim-3 on the cell surface would lead to a greater mTOR-inhibiting effect of the nanoconjugates. THP-1 cells were exposed to $100 \mathrm{nM}$ PMA for $24 \mathrm{~h}$ followed by $3 \mathrm{~h}$ exposure to the nanoconjugates (illustrated in Fig. 7). The same conditions as described above were

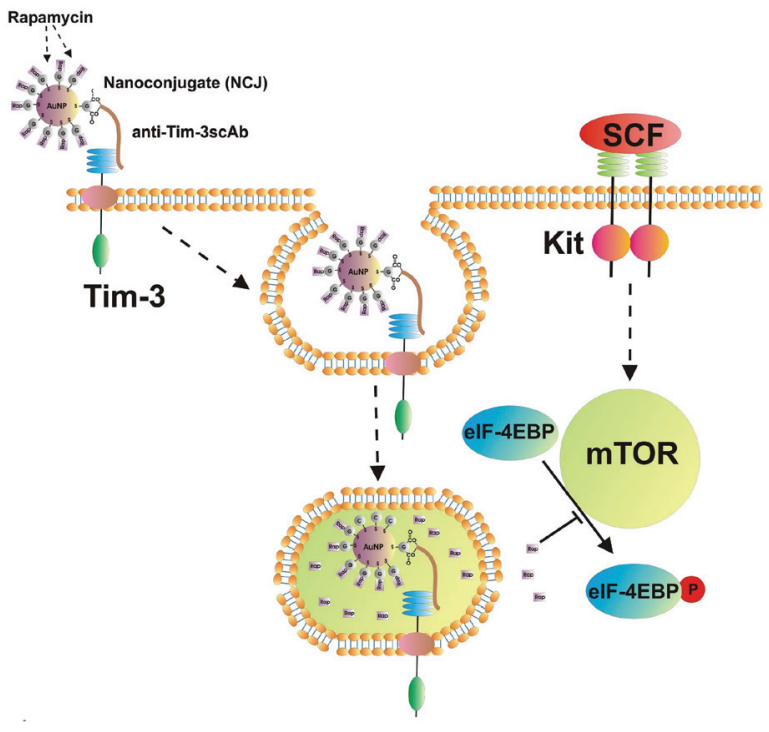

Fig. 5 Schematic representation of rapamycin delivery into a resting THP-1 human AML cell. The scheme shows SCF-induced activation of the mTOR pathway (in particular, mTOR-catalysed phosphorylation of the elF4E-BP). It also demonstrates the delivery path of rapamycin and inhibition of mTOR kinase activity.

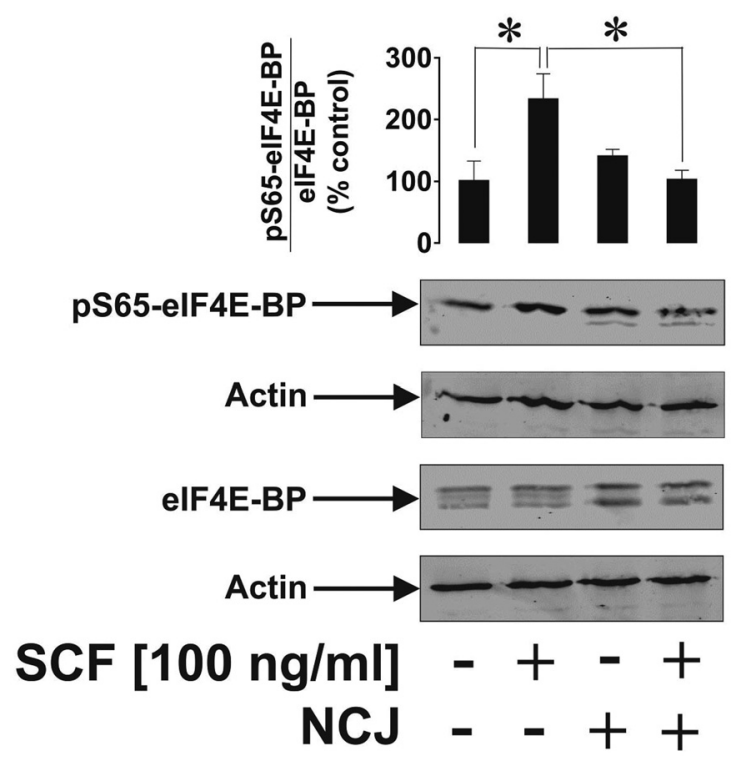

Fig. 6 Nanoconjugates prevent but do not abolish mTOR activity in undifferentiated THP-1 cells. Cells were pre-treated with NCJ for $1 \mathrm{~h}$ to allow for the delivery of maximally $200 \mathrm{nM}$ of rapamycin in the medium. Cells were then exposed for $2 \mathrm{~h}$ to $100 \mathrm{ng} \mathrm{ml}^{-1}$ of SCF or left without further treatment followed by the measurement of phospho-S65 and total elF4E-BP by western blot as described in the Materials and methods section. Images are from one experiment representative of three which gave similar results. Data show mean values \pm SEM of three independent experiments; * $p<0.05$.

applied. In this case NCJ completely abrogated the mTORdependent phosphorylation of eIF4E-BP (Fig. 8). Neither of the treatments affected cell viability. Interestingly, AuNPs carrying 


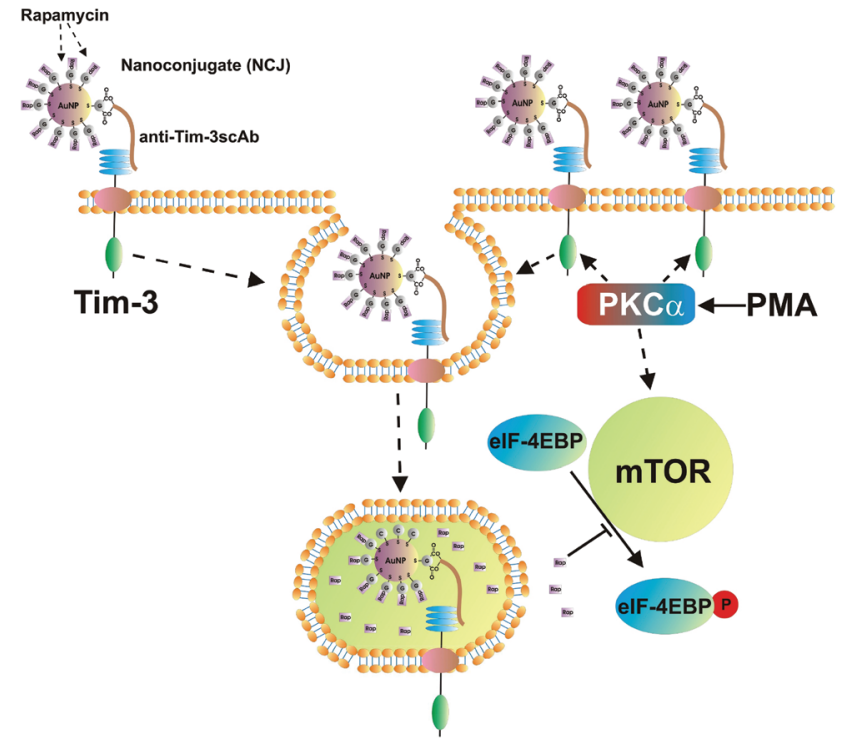

Fig. 7 Schematic representation of rapamycin delivery into a PMAdifferentiated THP-1 human AML cell. The scheme shows PMA-induced activation of the mTOR pathway in a PKC $\alpha$-dependent manner. It also demonstrates the delivery path of rapamycin and the subsequent inhibition of the mTOR kinase activity.

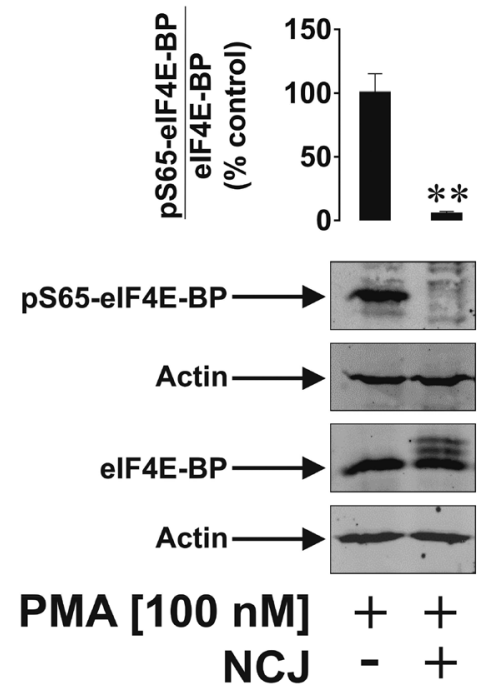

Fig. 8 Nanoconjugates attenuate mTOR activity in PMA-differentiated THP-1 cells. Cells were exposed for $24 \mathrm{~h}$ to $100 \mathrm{nM}$ PMA followed by $3 \mathrm{~h}$ of exposure to nanoconjugates. Images are from one experiment representative of four which gave similar results. Data show mean values \pm SEM of four independent experiments; ${ }^{* *} p<0.01$.

either anti-Tim-3-ScAb or rapamycin did not affect mTOR activity in either resting or PMA-treated THP-1 cells (data not shown).

Based on these results one could suggest that the successful inhibition of the mTOR-dependent phosphorylation of eIF4E-BP in THP-1 human AML cells depends on the amount of Tim-3 on the cell surface. Anti-Tim-3-ScAb molecules coupled with AuNPs interacted with THP-1 cells, which alike a
A

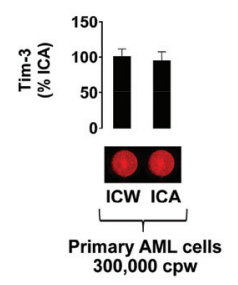

B

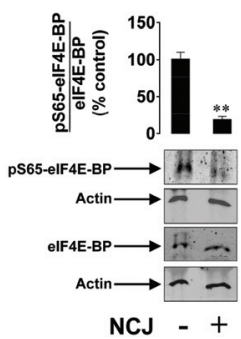

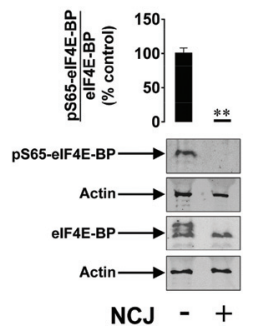

Fig. 9 Nanoconjugates attenuate mTOR activity in primary human AML cells. Tim-3 expression was mostly confined to the cell surface as measured by the in-cell assay and in-cell western analysis (A). Cells were exposed for 3 (B) or 24 (C) $h$ to nanoconjugates followed by western blot analysis of S65 phosphorylation of elF4E-BP. Images are from one experiment representative of four which gave similar results. Data show mean values \pm SEM of four independent experiments; ${ }^{* *} p<0.01$.

vast majority of blood cells can perform endocytosis. GSH was then cleaved by membrane-associated enzymes releasing rapamycin inside the target cell (otherwise, the effect will not be observed, since only free rapamycin is capable of inhibiting mTOR activity inside AML cells).

It is important to note that in order to achieve a complete abrogation of mTOR activity in THP-1 cells, the concentration of free rapamycin has to be at least $10 \mu \mathrm{M}$. The use of NCJ led to the same effect but at the rapamycin concentration 50 times lower than the one needed when using the drug in its free form.

The results obtained were validated using primary human AML cells. Tim-3 was clearly expressed on the cell surface as detected by the on cell assay vs. in cell western analysis. The cells appeared to be highly sensitive to the effects of nanoconjugates since the levels of phospho-S65-eIF4E-BP were significantly downregulated after $3 \mathrm{~h}$ of exposure to NCJs. Almost complete attenuation was observed after $24 \mathrm{~h}$ of exposure (Fig. 9).

\section{Conclusions}

Our results demonstrate that the targeted nanocarrier we have developed enables the delivery of otherwise toxic drugs into leukaemia cells with a high level of efficiency through Tim-3. Successful delivery depends on the cell surface expression of the target protein, which in our case was Tim-3. These nanoconjugates now require further studies using ex vivo and in vivo systems. This technology might also be considered for highly specific capturing of target cells that carry a surfacebased protein biomarker of interest.

\section{Conflicts of interest}

There are no conflicts of interest to declare. 


\section{References}

1 E. Vick and D. Mahadevan, Expert Opin. Invest. Drugs, 2016, 25, 1-16.

2 P. Romero, et al., Sci. Transl. Med., 2016, 8, 334 ps9, DOI: 10.1126/scitranslmed.aaf0685.

3 V. V. Sumbayev, I. M. Yasinska, C. P. Garcia, D. Gilliland, G. S. Lall, B. F. Gibbs, D. R. Bonsall, L. Varani, F. Rossi and L. Calzolai, Small, 2013, 9, 472-477.

4 B. F. Gibbs, I. M. Yasinska, L. Calzolai, D. Gilliland and V. V. Sumbayev, J. Biomed. Nanotechnol., 2014, 10, 1259-1266.

5 C. Pascual Garcia, V. V. Sumbayev, D. Gilliland, I. M. Yasinska, B. F. Gibbs, D. Mehn, L. Calzolai and F. Rossi, Sci. Rep., 2013, 3, 1326.

6 I. Gonçalves Silva, L. Ruegg, B. F. Gibbs, M. Bardelli, A. Fruewirth, L. Varani, S. Berger, E. Fasler-Kan and V. V. Sumbayev, OncoImmunology, 2016, 5, e1195535.

7 Y. Kikushige and T. Miyamoto, Int. J. Hematol., 2013, 98, 627-633.

8 I. Goncalves Silva, B. F. Gibbs, M. Bardelli, L. Varani and V. V. Sumbayev, Oncotarget, 2015, 6, 33823-33833.

9 A. Prokhorov, B. F. Gibbs, M. Bardelli, L. Ruegg, E. FaslerKan, L. Varani and V. V. Sumbayev, Int. J. Biochem. Cell Biol., 2015, 59, 11-20.

10 R. Coradeghini, S. Gioria, C. P. García, P. Nativo, F. Franchini, D. Gilliland, J. Ponti and F. Rossi, Toxicol. Lett., 2013, 217, 205-216.
11 C. Pabst, J. Krosl, I. Fares, G. Boucher, R. Ruel, A. Marinier, S. Lemieux, 4 Hébert and G. Sauvageau, Nat. Methods, 2014, 11, 436-442.

12 E. Fasler-Kan, N. Barteneva, S. Ketterer, K. Wunderlich, J. Huwyler, D. Gygax, J. Flammer and P. Meyer, Int. J. Interferon, Cytokine Mediator Res., 2010, 2, 127136.

13 R. Hussain, T. Javorfi and G. Siligardi, J. Synchrotron Radiat., 2012, 19, 132-135.

14 R. Hussain, T. Jávorfi and G. Siligardi, Compr. Chirality, 2012, 8, 438-448.

15 R. Hussain, K. Benning, D. Myatt, T. Javorfi, E. Longo, T. R. Rudd, B. Pulford and G. Siligardi, J. Synchrotron Radiat., 2015, 22, 862.

16 A. Midsonai, F. Wien, L. Kernya, Y.-H. Lee, Y. Goto, M. Réfrégiers and J. Kardos, Proc. Natl. Acad. Sci. U. S. A., 2015, 112, E3095-E3103.

17 F. Tietze, Anal. Biochem., 1969, 27, 502-522.

18 L. Calzolai, D. Gilliland, C. P. Garcìa and F. Rossi, J. Chromatogr. A, 2011, 1218, 4234-4239.

19 M. I. Setyawati, R. V. Kutty and D. T. Leong, Small, 2016, 12, 5601-5611.

20 I. Gonçalves Silva, I. M. Yasinska, S. S. Sakhnevych, W. Fiedler, J. Wellbrock, M. Bardelli, L. Varani, R. Hussain, G. Siligardi, G. Ceccone, S. M. Berger, Y. A. Ushkaryov, B. F. Gibbs, E. Fasler-Kan and V. V. Sumbayev, EBioMedicine, 2017, 22, 44-57. 